\title{
LES CLADOCÈRES \\ ET LEUR REPRODUCTION
}

\author{
Par M. KAJ BERG \\ Docteur ès philosophie, \\ assistant au Laboratoire d'hydrobiologie de l'Université de Copenhague à Hilleröd.
}

(Suite) (1)

\section{IV, - Nourriture}

Au point de vue du régime alimentaire, les Cladocères se répartissent cn deux groupes.

Le premier est constitué par les prédateurs qui se repaissent de bestioles vivantes. C'est le groupe le plus petit, car il comprend seulement deux familles : celles des Polyphémides et des Leptodorides. Leurs représentants ont une organisation correspondant à leur mode de nutrition : leurs membres ne sont pas lamelleux, mais armés de gniffes pour agripper les proies qui sont, par exemple, de petits Crustacés. Chez Leptodora (fig. 49), disposés en couronne constituant un piège, ils ont une grande aisance de mouvements, n'étant pas emprisonnés sous la carapace, et sont munis de pointes qui reliennont les victimes qui, ensuite, sont dépecées par les pièces buccales, pointues et dentées.

Le sccond groupe des Cladocères est celui des filtreurs, qui a la prépondérance comme rassemblant toutes les espèces n'appartenant pas aux deux familles susdésignées. Elles vivent aux dépens des menues particules alimentaires en suspension dans l'eau, consommant surtout des Algues, y compris celles si ténues qu'on ne peut les récolter avec le plus fin des filets à plancton, il faut pour les obtenir recourir à la centrifugation. Sont absorbés, en oulre, les menus débris de matière organique; toutes les espèces de Cladocères filtrcurs en font probablement plus ou moins grande consommation et, dans certains cas, s'en nourrissent presque exclusivement. En particulier les résidus de la vie organique tombant sur le fond des lacs servent de pâture aux Cladocères benthiques, notamment aux Macrothricides.

Le mécanisme du filtrage chez la Daphnie a élé bien étudiće par STorch (I924) ; l'appareil compliqué affecté à ce service aspire l'eau, retient les matières en suspension et les amène à la bouche. Quand on examine un

(

Quelques fautes d'impression sont à rectifier daṇs ce numéro:

P. 358 . - La figure devrait porter la mention : - l'aprìs O. Sroncm.

P. 36r. - A la jérende de la figure 45, lire : Drepanolrix et non Drepanoptrix.

1. 363. - $\Lambda$ la légende de la figure 5o, lire : Iliocryplus et non Hiocryplus. 
individu vivant, on distingue nettement, entre la tète et la queuc, ses membres ciliés (fig. 42). Animés d'un mouvement constant de va-et-vient au rythme de 200300 alternances par minute, ils jouent le rôle principal dans l'appel d'eau. Quand, au cours de leurs déplacements, les pattes arrivent à une certaine position, cette eau est aspirée dans l'espace existant entre elles. Puis la conlinuation du mouvement la contraint à passer au travers des franges de cils dont ces pattes sont bordées, pour être enfin expulsée. Les Algues et les débris retenus sont ensuite entraînés vers un canal, situé sur la face ventrale de l'animal, par lequel ils sont élevés jusqu'à la bouche sous l'action de quelques poils particulièrement développés de la seconde paire de membres. Parvenus à portée des mandibules, les aliments sont saisis par elles, broyés entre lcurs rugosilés, puis ingérés dans le pharynx d'où ils passent dans l'intestin.

Le travail de filtration s'opère très rapidement. E. Naumans (I92I) a observé le temps mis par quelques espèces à renouveler complètement le contenu de leur intestin. Il a constaté que cette opération demandait I5 minutes à Holopedium, Ceriodaphnia, Scapholeberis et Bosmina, 20 à 30 minutes à Daphnia et 60 minutes à Sida.

\section{V. - Fluctuations du peuplement}

A pêcher pendant un certain laps de temps des Cladocères dans un lac donné, on constate que ces animaux ne sont pas toujours pareillement abondants. A certains moments ils pullulent, passant alors par un maximum; à d'autres ils se raréfient et on a un minimum. En outre, il est des espèces qui ne se rencontrent pas d'un bout de l'année à l'autre, mais disparaissent pendant l'hiver; en d'autres termes, elles sont périodiques, alors que le reste est pérenne.

Parmi les premières citons : Leptodora Kindtii (fig. 49), Bythotrephes longimanus (fig. 48) et Diaphanosoma brachyurum (fig. 43) ; elles n'entrent que l'été dans la composition du plancton, leur abondance étant sujette à très amples variations. Ainsi, dans le lac de Frederiksborg, Diaphanosoma accuse un maximum en Juillet, à raison de 25 individus par litre d'eau contre 2 ou 2 quelcues semaines avant et après.

Pour les formes pérennes, quelques exemples donneront idée des écarts entre leurs hauts et leurs bas.

Daphnia magna, hôte exclusif des étangs peu profonds, à fond vaseux, offrant une abondante pâture, appartient généralement à cette catégorie, non toujours cependant. L'amplitude des maxima et minima, ainsi que leurs époques, varient beaucoup selon les lieux. Si grandes que soient les ressemblances entre deux nappes d'eau, il faut se rendre compte de l'action des petites différences de superficie, profondeur, alimentation en eau, teneur en matières organiques, etc., qui exercent leur influence sans répit tout le long de l'année. Néanmoins, il est assez aisé de dégager, dans leur ensemble, les lois qui régissent les mouvements de leurs populations en 
Daphnies. Durant la saison où une légère croûte de glace recouvre la surface liquide, l'espèce est, à l'ordinaire, faiblement représentée, se reproduisant ou non. Quand vient le printemps, l'air comme l'eau tiédissent et l'effectif augmente. Quand il s'est élevé jusqu'à un cerlain niveau, il peut, en quelques endroits, rester presque stationnaire durant tout l'été et la première partie de l'automne pour ne décroître qu'aux approches de l'hiver. Toutefois, la norme semble être qu'un maximum printanier est suivi d'un minimum estival, ce apsès quoi $\mathrm{D}$. magna foisonne à nouveau et présente un second maximum aulomnal, auquel succède la raréfaclion hivernale. Ces alternances comportent, bien entendu, des variantes locales pour ce qui regarde leurs dates et leurs amplitudes. Il arrive, par exemple, que la diminution, au cours de l'été, soit faiblement prononcée ou, au contraire, tcllement marquée que l'espèce subit comme une éclipse totale en Juillet pour ne plus reparaitre qu'en Septembre. Dans certains étangs, peu nombreux, elle se rencontre seulement durant quelques mois et il n'y a qu'un unique maximum se plaçant alors en été. Mais ce dernier cas est exceptionnel.

Une autre forme pérenne est Daphnia pulex, un des Cladocères les plus répandus. Contrairement à $D$. magna, elle fréquente des milicux très variés : mares de villages ou de fermes, ou encore voisines d'habitations, plus ou moins polluées, collecteurs de drainage, grands étangs, marais, parfois mème zone littorale des lacs où, cependant, on trouve plus ordinairement $D$. longispinc. Dans des milieux aussi différents, il est naturel que les mouvements de la population ne soient pas d'une rigoureuse uniformité. Au surplus, sur le même emplacement, s'observent parfois des différences considérables entre une année et les suivantes. Il est courant qu'on pêche $D$. pulex sous la glace au début de la saison froide, mais on n'a la certitude de sa pérennité que si sa présence est constatée en FévrierMars et $\Lambda$ vril, autrement dit durant la période précédant immédiatement celle du réchauffement printanier. On s'est rendu compte que $D$. pulex se maintient pendant l'hiver seulement dans quelques localités; qu'cn outre. pour cleux annécs consécutives, des différences inattendues se manifestent.

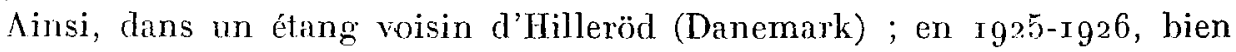
que congelé en surface durant trois à quatre mois, il n'a pas cessé d'héberger D. pulex; en r $9^{26-1} 9^{2} 7$, cette espèce fit défaul au cours d'un hiver bénin, l'étang n'ayant été pris que fort peu de temps. Quand pareille discontinuité s'observe, la réapparition, au printemps, a lieu à des dates et à des températures très variables. En Danemark, il advient qu'on rencontre de jeunes sujpts dès les premiers jours de Mars, alors que la masse de l'eau est encore très voisine de $o^{\circ} \mathrm{C}$., tandis qu'ils sont introuvables, ailleurs, mème en Avril, quand la température atteint 8 à $\iota^{\circ} \mathrm{C}$.; il faut attendrc lcur apparition jusqu'au début de Mai. Entre ces cas cxtrêmes s'intercalent tous ceux intermćdiaires.

Qu'clle ait subsisté ou non pendant l'hiver, D. pulex se multiplie abondamment an printemps. Au bout de quelques semaines elle pullule. Cette 
exhaustion du peuplement s'observe partout entre le début de Mars et la fin de Mai, mais ensuite les fluctuations sont fort variables.

Lc maximum printanier peut ètre suivi d'une lente diminulion aboutissant, finalement, à la disparition de l'espèce. Elle se produit, naturellement, dans les mares qui s'assèchcnt, mais encore dans les élangs qui, fout en conservant de l'eau au plus fort de l'été, sont de profondeur très faible. Quand $D$. pulex fail délaut cntre Juin el Août, il advient qu'elle manque tout le reste de l'année, même quand l'eau revient à l'automne ; on n'observe alors qu'un seul maximum, qui se place au printemps. Mais, sur d'autres emplacements, il y a réapparition avec prolifération abondante consécutive et par suite, un second maximum, à l'aulomne, éventucllement aussi marqué que le premier.

Dans les étangs où les pertes par évaporation ne sont pas trop considérables en été, $D$. pulex se rencontre cncore aux mois de Juin, Juillet, Août ; mais raréfiée. Dans ces conditions, on enregistre encore un maximum printanier et un autre automnal.

Ces deux modes d'alternance peuvent se constater successivement dans une même localité.

Finalement, comme forme pérenne des lacs, citons Daphnia cucullata. Le nombre de ses représentants est très réduit pendant l'hiver, si faible parfois qu'on ne les décèle pas sans difficulté. Au printemps, le contingent augmente et, vers la fin de l'été, atteint son maximum unique pour diminuer ensuite brusquement. Signalons qu'au moment du maximum nous avons trouvé, par litre d'eau, de I 75 (rg25) à 225 sujets (rg26).

Au reste, le foisonnement des Cladocères lors de leur apogée est souvent fantastique. Aux moments où elle surabonde, $D$. pulex en arrive à communiquer une teinte brune à l'eau des étangs.

Ces animalcules ne manifestent pas une tendance marquée à former des essaims, encore que semblables rassemblements s'observent quelquefois. Ainsi, avons-nous vu un jour un étang, de quelque distance du bord, préscnter des sortes de grandes tachos d'un jaune rougeâtre, notamment à l'entour de toulfes confluentes de Glyceria. Un examen attentif révéla que cette apparence était due à des myriades de $D$. pulex. Il arrive aussi que $D$. magna forme de grands bancs.

Quelques indications, s'ajoutant à celles déjà fournies, donneront idée de re que peut ctre le maximum des Cladocères. Chydorus sphaericus, hôte fréquent de la zone littorale des lacs, peut aussi devenir un organismo du plancton pólagique. Comme tel, il y a été rencontré à la dose de 500 individus par litre d'eau. Mais ce nombre est encore dépassé par Bosmina longispina, avec 950 individus, ce qui en fait presque un par centimètre cube ! 